\title{
Buried Compressional Line Source in a Halfspace with an Irregular Boundary.
}

\author{
By \\ Iya ABubaKar \\ St. Catherine's College, Cambridge
}

\begin{abstract}
Summary
By an application of the method of small perturbations an approximate solution of the problem of a compressional line source in a halfspace whose free surface is irregular is obtained in an integral form, on the assumption that the surface slope and the magnitude of the irregularities are small. The resulting integrals for the secondary waves whose amplitudes are proportional to the depth of the surface corrugation are evaluated approximately by the method of multiple steepest descent. Two particular surfaces are considered: a sinusoidal surface and a symmetrical curvilinear surface in the form of a basin. It is found that if the ratio of the wavelength of the incident wave to the halfwidth of the basin is small the secondary waves are reflected in the directions of specular reflection and if the ratio is large, the waves appear to come from the basin. With the sinusoidal surface, if the ratio of surface wavelength to the length of the incident waves is large the secondary waves are reflected in the specular directions and if the ratio is small then the surface acts as a grating, the secondary waves being reflected from the troughs of the surface. The amplitudes of the secondary waves are inversely proportional to the square root of range and the deeper the source the smaller the amplitudes.
\end{abstract}

\section{§1. Introduction}

Earthquake generated seimic waves encounter on their paths mountains and basins, salt and ore bodies and corrugated strata in fold regions, and such structures, no cloubt, affect the transmission of at least the short period waves. Seismic prospecting is widely carried out in regions of complex geological structures whose reflecting and refracting capacities are nonspecular in nature. Hence the study of the effect of the irregularity of seismic waves is of some practical importance.

SkURIDIN (1957), SAto (1955, 1956), GILBERT and KNopoff (1960) and ABubaKar (1962), among others, have studied the problem of the scattering of plane elastic waves at irregular surfaces using various approximating techniques.

In the present paper the problem of a buried compressional line source in a halfspace whose free surface is irregular is investigated using a modification of RICE's (1951) perturbation method of approximation.

\section{§2. Formal Solution}

Choose a rectangular Cartesian system of coordinates Oxyz. We confine ourselves to the two-dimensional case of the problem with the waves polarized in the $x z$-plane. Let the cross-section of the bounding surface in the plane $y=0$ be

$$
z=\varepsilon f(x),
$$

where $\varepsilon$ is a small constant and the function $f(x)$ and its first derivative are continuous and bounded everywhere. It is assumed that the slope of the surface and the ratio of the maximum amplitude of the surface corrugation to the wavelength of the incident wave are everywhere small.

The homogeneous isotropic elastic medium in which the waves are propagated occupies the region $-\infty<x, y<+\infty, z \geq \varepsilon f(x)$. Let $\rho$ be the density of the medium, and $\lambda, \mu$ its Lame's parameters. The $z$-axis is directed vertically into the medium.

Let $\phi, \phi$ be the displacement potentials associated with $P$ and $S V$ waves respectively. 
They satisfy the familiar scalar wave equations, namely

$$
\begin{aligned}
& \nabla^{2} \phi=\frac{1}{\alpha^{2}} \frac{\partial^{2} \phi}{\partial t^{2}}, \\
& \nabla^{2} \phi=\frac{1}{\beta^{2}} \frac{\partial^{2} \psi}{\partial t^{2}},
\end{aligned}
$$

where $\alpha, \beta$ are the propagation velocities of $P$ and $S$ waves respectively in the medium, and are given by

$$
\alpha^{2}=(\lambda+2 \mu) / \rho, \quad \beta^{2}=\mu / \rho .
$$

Let $n, s$ be the unit vectors normal and tangential to the curve $\varepsilon f(x)$. Then if $p_{z z}$, $p_{z x}, \cdots, p_{n n}, p_{n s}$ denote the components of stress referred to the $x z$ and $n s$ axes respectively they are related by:

$$
\left.\begin{array}{l}
p_{n n}=\left(p_{z z}+\varepsilon^{2} f^{\prime 2} p_{x x}-2 \varepsilon f^{\prime} p_{x x}\right) /\left(1+\varepsilon^{2} f^{\prime 2}\right), \\
\left.p_{n s}=\left[\left(p_{x x}-p_{z z}\right) \varepsilon f^{\prime}-\left(1-\varepsilon^{2} f^{\prime 2}\right) p_{x z}\right)\right] /\left(1+\varepsilon^{2} f^{\prime 2}\right),
\end{array}\right\}
$$

where $\varepsilon f^{\prime}=d z / d x$, and

$$
\left.\begin{array}{l}
p_{z z}=\lambda \nabla^{2} \phi+2 \mu\left(\phi_{z z}+\phi_{x z}\right), \\
p_{x x}=\lambda \nabla^{2} \phi+2 \mu\left(\phi_{x x}+\phi_{x z}\right), \\
p_{z x}=\mu\left(2 \phi_{x z}-\phi_{z z}+\phi_{x x}\right) .
\end{array}\right\}
$$

Let there be a source of disturbance at the point $(0, h)$ in the medium generating $P$ (or $S V)$ waves whose time variation is $e^{i \omega t}$. Then the displacement potential in the medium can be represented by:

$$
\begin{aligned}
\phi= & -a e^{i \omega t} \int_{-\infty}^{\infty} \frac{1}{v_{1}} \exp \left[-v_{1}|h-z|-i \zeta x\right] d \zeta \\
& +e^{i \omega t} \int_{-\infty}^{\infty} A(\zeta) \exp \left(-v_{1} z-i \zeta x\right) d \zeta, \\
\phi= & -b e^{i \omega t} \int_{-\infty}^{\infty} \frac{1}{v_{2}} \exp \left[-v_{2}|h-z|-i \zeta x\right] d \zeta \\
& +e^{i \omega t} \int_{-\infty}^{\infty} B(\zeta) \exp \left(-v_{2} z-i \zeta x\right) d \zeta .
\end{aligned}
$$

where since $\phi, \phi$ satisfy equations (2.2) and (2.3) respectively we have

$$
\left.\begin{array}{ll}
v_{1}^{2}=\zeta^{2}-k_{1}{ }^{2}, & v_{2}{ }^{2}=\zeta^{2}-k_{2}{ }^{2}, \\
k_{1}=\omega / \alpha, & k_{2}=\omega / \beta,
\end{array}\right\}
$$

and we define the radicals such that $R e v_{1} \geq 0$, $R e v_{2} \geq 0$. This is to ensure that the potentials satisfy Sommerfeld's radiation condition at infinity, that is, they remain bounded at infinite depth.

If the initial source is of $P$-type we put $a=1, b=0$ in (2.7) and (2.8) and if it is of $S V$-type we put $a=0, b=1$.

The first terms of (2.7) and (2.8) represent the initial disturbance in an unbounded medium. That form of representation of a harmonic line source potential goes back to LAMB (1904). The second terms represent the effect of the presence of the boundary. They consist of a superposition of (i) plane waves propagating downwards in all directions in the medium and (ii) waves whose energy is confined to the neighbourhood of the surface. These have various wave numbers in the $x$-direction ranging from $k_{1,2}$ to $\infty$. But as the wave numbers tend to infinity the amplitudes tend to zero. Thus the second terms represent the scattered reflected waves. The functions $A(\zeta), B(\zeta)$ give the relative amplitude distribution of the superposed scattered waves and we may refer to them as the spectral densities. They are determined from the bounclary conditions.

\section{§3. Boundary Conditions}

The boundary condition is that the surface is stress-free which requires that

$$
p_{n n}=p_{n s}=0 \text { on } z \cdots \varepsilon f \text {. }
$$

From (2.5), neglecting terms of $O\left(\varepsilon^{2}\right)$, this becomes

$$
\left.\begin{array}{c}
p_{z z}-2 \varepsilon f^{\prime} p_{z x}=0, \\
\left(p_{z x}-p_{x x}\right) \varepsilon f^{\prime}+p_{z x}=0
\end{array}\right\} \text { on } z=\varepsilon f .
$$

On expressing the above equations (3.2) in terms of the displacement potentials using (2.6) and by substituting the assumed displacement potentials (2.7) and (2.8) in the result we obtain, on omitting the time factor $e^{i \omega t}$ (to be reinserted when required),

$$
\begin{aligned}
& a \int_{-\infty}^{\infty} \frac{\lambda k_{1}^{2}-2 \mu v_{1}^{2}}{v_{1}} \exp \left[-v_{1}(h-\varepsilon f)-i \zeta x\right] d \zeta \\
& -\int_{-\infty}^{\infty}\left(\lambda k_{1}^{2}-2 \mu v_{1}^{2}\right) A(\zeta) \exp \left(-v_{1} \varepsilon f-i \zeta x\right) d \zeta \\
& \quad+2 i \mu b \int_{-\infty}^{\infty} \zeta \exp \left[-v_{2}(h-\varepsilon f)-i \zeta x\right] d \zeta \\
& \quad+2 i \mu \int_{-\infty}^{\infty} v_{2} \zeta B(\zeta) \exp \left(-v_{2} \varepsilon f-i \zeta x\right) d \zeta \\
& \quad-2 \mu \varepsilon f^{\prime}\left\{2 i a \int_{-\infty}^{\infty} \zeta \exp \left[-v_{1}(h-\varepsilon f)-i \zeta x\right] d \zeta\right.
\end{aligned}
$$


$+2 i \int_{-\infty}^{\infty} \zeta v_{1} A(\zeta) \exp \left(-v_{1} \varepsilon f-i \zeta x\right) d \zeta$

$+b \int_{-\infty}^{\infty} \frac{v_{2}{ }^{2}+\zeta^{2}}{v_{2}{ }^{2}} \exp \left[-v_{2}(h-\varepsilon f)-i \zeta x\right] d \zeta$

$\left.-\int_{-\infty}^{\infty}\left(v_{2}^{2}+\zeta^{2}\right) B(\zeta) \exp \left(-v_{2} \varepsilon f-i \zeta x\right) d \zeta\right\}=0$
These equations hold only if $h>|\varepsilon f|$ for all $x$. This is readily seen from the form of the potentials (2.7) and (2.8). The two integral equations (3.3) and (3.4) determine $A(\zeta), B(\zeta)$.

\section{$\S 4$. Perturbation Solution}

It is impossible to extract the spectral densities $A(\zeta), B(\zeta)$ from the equations (3.3) and (3.4) for arbitrary function $f(x)$ without approximating. We use a perturbation method. We assume that $A(\zeta), B(\zeta)$ can be expanded into sums of terms which are of the order of powers of $\varepsilon$, and that the first two terms give their aprroximate values. Thus

$$
\left.\begin{array}{l}
A(\zeta)=A_{0}(\zeta)+\varepsilon A_{1}(\zeta)+\varepsilon^{2} A_{2}(\zeta)+\cdots, \\
B(\zeta)=B_{0}(\zeta)+\varepsilon B_{1}(\zeta)+\varepsilon^{2} B_{2}(\zeta)+\cdots,
\end{array}\right\}
$$

where $A_{j}(\zeta), B_{j}(\zeta)(j=0,1,2, \cdots)$ are of $O\left(\varepsilon^{0}\right)$. Inserting the series representation of $A(\zeta)$, $B(\zeta),(4.1)$ into the integral equations (3.3) and (3.4) and expanding the exponential factors whose exponents are functions of $\varepsilon$, retaining only terms involving $\varepsilon$, we have:

$$
\begin{aligned}
a \int_{-\infty}^{\infty} & \frac{\lambda k_{1}^{2}-2 \mu v_{1}^{2}-4 \mu i \varepsilon \zeta v_{1} f^{\prime}}{v_{1}}\left(1+v_{1} \varepsilon f+\cdots\right) \exp \left(-v_{1} h-i \zeta x\right) d \zeta \\
& +\int_{-\infty}^{\infty}\left[\left(A_{0}+\varepsilon A_{1}+\cdots\right)\left(2 \mu v_{1}^{2}-\lambda k_{1}^{2}-4 i \mu \varepsilon \zeta v_{1} f^{\prime}\right)\left(1-v_{1} \varepsilon f+\cdots\right) e^{-i \zeta^{x}}\right] d \zeta \\
& +2 \mu \int_{-\infty}^{\infty}\left\{\left(B_{0}+\varepsilon B_{1}+\cdots\right)\left[i \zeta v_{2}+\left(v_{2}^{2}+\zeta^{2}\right) \varepsilon f^{\prime}\right]\left(1-v_{2} \varepsilon f+\cdots\right) e^{-i \zeta^{x}}\right\} d \zeta \\
& +2 \mu b \int_{-\infty}^{\infty} \frac{i v_{2} \zeta-\varepsilon f^{\prime}\left(v_{2}^{2}+\zeta^{2}\right)}{v_{2}}\left(1+v_{2} \varepsilon f+\cdots\right) \exp \left(-v_{2} h-i \zeta x\right) d \zeta=0 \\
2 a \int_{-\infty}^{\infty} & \frac{\varepsilon f^{\prime}\left(\zeta^{2}+v_{1}^{2}\right)-i v_{1} \zeta}{v_{1}}\left(1+v_{1} \varepsilon f+\cdots\right) \exp \left(-v_{1} h-i \zeta x\right) d \zeta \\
& -2 \int_{-\infty}^{\infty}\left\{\left(A_{0}+\varepsilon A_{1}+\cdots\right)\left[\varepsilon f^{\prime}\left(v_{1}^{2}+\zeta^{2}\right)+i v_{1} \zeta\right]\left(1-v_{1} \varepsilon f+\cdots\right) e^{-i \zeta x}\right\} d \zeta \\
& -\int_{-\infty}^{\infty}\left[\left(B_{0}+\varepsilon B_{1}+\cdots\right)\left(4 i \zeta v_{2} \varepsilon f^{\prime}-v_{2}^{2}-\zeta^{2}\right)\left(1-v_{2} \varepsilon f+\cdots\right) e^{-i \zeta x}\right] d \zeta \\
& -b \int_{-\infty}^{\infty} \frac{4 i \zeta v_{2} \varepsilon f^{\prime}+v_{2}^{2}+\zeta^{2}}{v_{2}}\left(1+v_{2} \varepsilon f+\cdots\right) \exp \left(-v_{2} h-i \zeta x\right) d \zeta=0 .
\end{aligned}
$$

To determine $A_{0}, B_{0}, A_{1}, B_{1}$ we collect terms of $O\left(\varepsilon^{0}\right), O\left(\varepsilon^{1}\right)$ and solve the resulting simul. taneous equations. Equating terms of $O\left(\varepsilon^{0}\right)$ and $O\left(\varepsilon^{1}\right)$ separately to zero we obtain:

$O\left(\varepsilon^{0}\right)$ : 


$$
\begin{aligned}
& a \int_{-\infty}^{\infty} \frac{\lambda k_{1}^{2}-2 \mu v_{1}^{2}}{v_{1}} \exp \left(-v_{1} h-i \zeta x\right) d \zeta+\int_{-\infty}^{\infty} A_{0}\left(2 \mu v_{1}^{2}-\lambda k_{1}^{2}\right) e^{-i \zeta x} d \zeta \\
& \quad+2 i \mu b \int_{-\infty}^{\infty} \zeta \exp \left(-v_{2} h-i \zeta x\right) d \zeta+2 \mu i \int_{-\infty}^{\infty} B_{0} \zeta v_{2} e^{-i \zeta x} d \zeta=0, \\
& 2 i \int_{-\infty}^{\infty} \zeta \exp \left(-v_{1} h-i \zeta x\right) d \zeta+2 i \int_{-\infty}^{\infty} \zeta v_{1} A_{0} e^{-i \zeta^{x}} d \zeta \\
& \quad+b \int_{-\infty}^{\infty} \frac{v_{2}^{2}+\zeta^{2}}{v_{2}} \exp \left(-v_{2} h-i \zeta x\right) d \zeta-\int_{-\infty}^{\infty}\left(v_{2}^{2}+\zeta^{2}\right) B_{0} e^{-i \zeta x} d \zeta=0 .
\end{aligned}
$$

$O\left(\varepsilon^{1}\right)$ :

$$
\begin{aligned}
& a \int_{-\infty}^{\infty}\left[f\left(\lambda k_{1}^{2}-2 \mu v_{1}^{2}\right)-4 \mu i \zeta f^{\prime}\right] \exp \left(-v_{1} h-i \zeta x\right) d \zeta \\
& +2 \mu b \int_{-\infty}^{\infty} \frac{i \zeta v_{2}{ }^{2} f-\left(v_{2}{ }^{2}+\zeta^{2}\right) f^{\prime}}{v_{2}} \exp \left(-v_{2} h-i \zeta x\right) d \zeta \\
& -\int_{-\infty}^{\infty}\left\{\left\{\left(2 \mu v_{1}^{2}-\lambda k_{1}^{2}\right) f+4 i \mu \zeta f^{\prime}\right] v_{1} A_{0}-\left(2 \mu v_{1}^{2}-\lambda k_{1}^{2}\right) A_{1}\right\} e^{-i \zeta^{x}} d \zeta \\
& -2 \mu \int_{-\infty}^{\infty}\left\{\left[i \zeta v_{2}^{2} f-\left(v_{2}^{2}+\zeta^{2}\right) f^{\prime}\right] B_{0}-i \zeta v_{2} B_{1}\right\} e^{-i \zeta^{x}} d \zeta=0, \\
& 2 a \int_{-\infty}^{\infty} \frac{\left(v_{1}^{2}+\zeta^{2}\right) f^{\prime}-i \zeta v_{1}^{2} f}{v_{1}} \exp \left(-v_{1} h-i \zeta x\right) d \zeta \\
& +b \int_{-\infty}^{\infty}\left[4 i \zeta f^{\prime}+\left(v_{2}^{2}+\zeta^{2}\right) f\right] \exp \left(-v_{2} h-i \zeta x\right) d \zeta \\
& -2 \int_{-\infty}^{\infty}\left\{\left[\left(v_{1}^{2}+f^{2}\right) f^{\prime}-i \zeta v_{1}{ }^{2} f\right] A_{0}+i v_{1}{ }^{2} \zeta A_{1}\right\} e^{-i \zeta^{\mu}} d \zeta \\
& -\int_{-\infty}^{\infty}\left\{\left[4 i \zeta f^{\prime}+f\left(v_{2}^{2}+\zeta^{2}\right)\right] v_{2} B_{0}-B_{1}\left(v_{2}^{2}+\zeta_{2}\right)\right\} e^{-i \zeta^{x}} d \zeta=0 .
\end{aligned}
$$

These integral equations can be solved for $A_{0}, B_{0}, A_{1}, B_{1}$. Thus far the solution holds for either compressional or distortional source of disturbance. We proceed to study the case of compressional disturbance.

\section{$\S$ 5. Initial $P$ Source}

To obtain the solution for an irrotational source we put $a=1, b=0$ in the equations (4.4)(4.7). Hence from (4.4) and (4.5) we obtain two integral equations which can be inverted by the Fourier inversion formula yielding

$$
\left.\begin{array}{c}
\left(\lambda k_{1}^{2}-2 \mu v_{1}^{2}\right) A_{0}-2 i \mu \zeta v_{2} B_{0}=\frac{\left(\lambda k_{1}^{2}-2 \mu v_{1}^{2}\right)}{v_{1}} \exp \left(-v_{1} h\right), \\
2 i \zeta v_{1} A_{0}-\left(v_{2}^{2}+\zeta^{2}\right) B_{0}=-2 i \zeta e^{-v_{1} h}
\end{array}\right\}
$$

Solving these equations yields:

$$
\left.\begin{array}{l}
A_{0}(\zeta)=\left[\left(\lambda k_{1}^{2}-2 \mu v_{1}^{2}\right)\left(v_{2}^{2}+\zeta^{2}\right)-4 \mu \zeta^{2} v_{1} v_{2}\right] e^{-v_{1} h} / v_{1} \Delta_{0}, \\
B_{0}(\zeta)=4 i \zeta\left(\lambda k_{1}^{2}-2 \mu v_{1}^{2}\right) e^{-v_{1} h} / \Delta_{0},
\end{array}\right\}
$$

where

$$
\Delta_{0}=\left(\lambda k_{1}{ }^{2}-2 \mu v_{1}{ }^{2}\right)\left(v_{2}{ }^{2}+\zeta^{2}\right)+4 \mu \zeta^{2} v_{1} v_{2} .
$$


Inserting the expressions (5.2) for $A_{0}, B_{0}$ into (2.7) and (2.8) we obtain the zero approximation potentials for the reflected waves as:

$$
\left.\begin{array}{l}
\phi_{0}=\int_{-\infty}^{\infty} \frac{\left(\lambda k_{1}^{2}-2 \mu v_{1}^{2}\right)\left(v_{2}^{2}+\zeta^{2}\right)-4 \mu \zeta^{2} v_{1} v_{2}}{v_{1} \Delta_{0}} \exp \left[-v_{1}(h+z)-i \zeta x\right] d \zeta, \\
\phi_{0}=4 i \int_{-\infty}^{\infty} \frac{\zeta\left(\lambda k_{1}^{2}-2 \mu v_{1}^{2}\right)}{\Delta_{0}} \exp \left[-v_{1} h-v_{2} z-i \zeta x\right] .
\end{array}\right\}
$$

These are the displacement potentials which are independent of the curvature of the surface. Expressions (5.4) can be identified as the formal solution of the problem of the disturbance due to a burried line source of $P$ type in an elastic halfspace. Lapwood (1949) has given a full discussion of these potentials and we turn our attention to terms of $O(\varepsilon)$ which represent the first order correction terms due to the curvature of the surface.

In equations (4.6) and (4.7) we put $a=1, b=0$ and insert the values of $A_{0}, B_{0}$ given by (5.2). Inverting the resulting equations and simplifying them we obtain

$$
\begin{aligned}
& \left(2 \zeta^{2}-k_{2}^{2}\right) A_{1}+2 i \zeta v_{2} B_{1} \\
& =\frac{1}{\pi} \int_{-\infty}^{\infty} e^{-i \zeta x} d x \int_{-\infty}^{\infty} \frac{\left(2 \zeta^{2}-k_{2}^{2}\right)}{\Delta}\left[\left(2 \zeta^{2}-k_{2}{ }^{2}\right)^{2}-4 \zeta^{2} v_{2}^{2}\right] f \exp \left(-v_{1} h-i \zeta x\right) d \zeta \\
& \equiv L(\zeta), \quad \text { say, } \\
& -2 i \zeta v_{1} A_{1}+\left(2 \zeta^{2}-k_{2}^{2}\right) B_{1} \\
& =\int_{-\infty}^{\infty} e^{-i \zeta x} d x \int_{-\infty}^{\infty}\left[\left\{i \zeta v_{2} f\left[\left(2 \zeta^{2}-k_{2}^{2}\right)^{2}-4 \zeta^{2} v_{1}^{2}\right]+4 v_{2} \zeta^{2}\left(k_{2}{ }^{2}-k_{1}{ }^{2}\right) f^{\prime}\right\} / \Delta\right] \exp \left(-v_{1} h-i \zeta x\right) d \zeta \\
& \equiv M(\zeta), \quad \text { say, }
\end{aligned}
$$

where $\Delta$ is given by

$$
\Delta=\left(2 \zeta^{2}-k_{2}^{2}\right)^{2}-4 \zeta^{2} v_{1} v_{2}
$$

Solving the simultaneous equations (5.5) and (5.6) we obtain

$$
\left.\begin{array}{l}
A_{1}(\zeta)=\left[\left(2 \zeta^{2}-k_{2}^{2}\right) L(\zeta)-2 i \zeta v_{2} M(\zeta)\right] / \Delta(\zeta), \\
B_{1}(\zeta)=\left[2 i \zeta v_{1} L(\zeta)+\left(2 \zeta^{2}-k_{2}^{2}\right) M(\zeta)\right] / \Delta(\zeta) .
\end{array}\right\}
$$

Inserting (5.8) into the expressions for the potentials of the scattered waves (2.7) and (2.8) we obtain the displacement potentials of the reflected waves whose amplitudes are of $O(\varepsilon)$ as:

$$
\left.\begin{array}{l}
\phi=\varepsilon \int_{-\infty}^{\infty} A_{1}(\zeta) \exp \left(-v_{1} z-i \zeta x\right) d \zeta \\
\phi=\varepsilon \int_{-\infty}^{\infty} B_{1}(\zeta) \exp \left(-v_{2} z-i \zeta x\right) d \zeta
\end{array}\right\}
$$

If we substitute the values of $A_{1}(\zeta)$ and $B_{1}(\zeta)$ into (5.9) we obtain triple integrals which cannot be evaluated in closed form and they have to be approximated. In geophysical problems approximate evaluation of integrals are effected, in general, by two methods: along steepest descent or stationary phase paths and along Sommerfeld contours. Each of these has its merits and demerits. The steepest descent and the stationary phase methods give fairly accurate travel times and amplitudes and also they give an indication of the distances at which the various pulses first appear. But they are suitable for only large frequencies so that the exponential factor of the integrand oscillates more rapidly compared with the 
changes of its multiplier. With the Sommerfeld contour the frequency needs not to be large and so the time variation of the source can be generalized. Moreover it brings out all the pulses (or waves) which appear. This makes it particularly suitable for plane waves.

So the triple integrals can be evaluated by successive approximations over each variable, taking into account of the contributions from branch cuts and poles. An alternative approach is the multi-dimensional steepest descent approximation developed by VAN der PoLe and BREMMER (1937). We will restrict our analysis to the latter method.

\section{§6. Steepest Descent Approximation}

In order to take into account the effect of the variation of $f(x)$ on the phases of the integrals we write $f(x)=e^{\log f(x)}$ and $f^{\prime}(x)=\left[f^{\prime}(x) / f(x)\right] e^{\log f(x)}$. Writing $\tau$ and $\eta$ for $\zeta$ and $x$ respectively in the integrals (5.5) and (5.6) and making use of (5.8) and (5.9) we obtain:

$$
\begin{aligned}
& \phi=\varepsilon \iiint_{-\infty}^{\infty} G_{1}(\eta, \zeta, \tau) \exp \left[g_{1}(\eta, \zeta, \tau)\right] d \eta d \zeta d \tau, \\
& \phi=\varepsilon \iiint_{-\infty}^{\infty} G_{2}(\eta, \zeta, \tau) \exp \left[g_{2}(\eta, \zeta, \tau)\right] d \eta d \zeta d \tau,
\end{aligned}
$$

where

$$
\begin{aligned}
& g_{1}(\eta, \zeta, \tau)=\log f(\eta)-v_{1} z-\bar{v}_{1} h-i \tau \eta-i \zeta(x-\eta), \\
& g_{2}(\eta, \zeta, \tau)=\log f(\eta)-v_{2} z-\bar{v}_{1} h-i \tau \eta-i \zeta(x-\eta), \\
& v_{1,2}=\left(\zeta^{2}-k_{1,2}\right)^{1 / 2} \text { and } \bar{v}_{1,2}=\left(\tau^{2}-k_{1,2^{2}}\right)^{1 / 2} \text {, } \\
& G_{1}(\eta, \zeta, \tau)=\left(2 \zeta^{2}-k_{2}^{2}\right)\left(2 \tau^{2}-k_{2}^{2}\right)\left[\left(2 \tau^{2}-k_{2}^{2}\right)^{2}-4 \tau^{2} \bar{v}_{2}^{2}\right] / \pi \Delta(\zeta) \Delta(\tau) \\
& -4 i \zeta v_{2}\left\{i \tau \bar{v}_{2}\left[\left(2 \tau^{2}-k_{2}{ }^{2}\right)^{2}-4 \tau^{2} \bar{v}_{1}{ }^{2}\right]+4 \bar{v}_{2} \tau^{2}\left(k_{2}{ }^{2}-k_{1}{ }^{2}\right) f^{\prime} / f\right\} / \pi \Delta(\zeta) \Delta(\tau), \\
& G_{2}(\eta, \zeta, \tau)=2 i \zeta v_{1}\left(2 \tau^{2}-k_{2}^{2}\right)\left[\left(2 \tau^{2}-k_{2}^{2}\right)^{2}-4 \tau^{2} \bar{v}_{2}^{2}\right] / \pi \Delta(\zeta) \Delta(\tau) \\
& +2\left(2 \zeta^{2}-k_{2}{ }^{2}\right)\left\{i \tau \bar{v}_{2}\left[\left(2 \tau^{2}-k_{2}{ }^{2}\right)^{2}-4 \tau^{2} \bar{v}_{1}^{2}\right]+4 \tau^{2} \bar{v}_{2}\left(k_{2}{ }^{2}-k_{1}{ }^{2}\right) f^{\prime} / f\right\} / \pi \Delta(\zeta) \Delta(\tau) .
\end{aligned}
$$

We consider the integral (6.1) first. The three-dimensional saddle point, $\left(\eta_{0}, \zeta_{0}, \tau_{0}\right)$ say, is given by

These give

$$
\frac{\partial g_{1}}{\partial \eta}=\frac{\partial g_{1}}{\partial \zeta}=\frac{\partial g_{1}}{\partial \tau}=0
$$

$$
\begin{aligned}
& f^{\prime}(\eta) / f(\eta)-i \tau+i \zeta=0, \\
& -z \zeta / v_{1}-i(x-\eta)=0, \\
& -h \tau \sqrt{\bar{v}_{1}}-i \eta \quad=0 .
\end{aligned}
$$

Then the steepest descent approximation to the integral is:

$$
\phi=\varepsilon \frac{(i \sqrt{2 \pi})^{3}}{\Delta^{1 / 2}} G_{1}\left(\eta_{0}, \zeta_{0}, \tau_{0}\right) \exp \left[g_{1}\left(\eta_{0}, \zeta_{0}, \tau_{0}\right)\right],
$$

where $\Delta$ is Hesse's determinant of order three of $g_{1}(\eta, \zeta, \tau)$ evaluated at the saddle point.

Thus the approximate amplitude and phase of the disturbance at the point $(x, z)$ is given by (6.12). This is exact for short wavelengths, for which geometrical ray theory is, in general, applicable. 
From (6.10) and (6.11) we obtain,

$$
\tau_{0}=k_{1} \eta_{0}\left(h^{2}+\eta_{0}\right)^{1 / 2}, \quad \zeta_{0}=k_{1}\left(x-\eta_{0}\right) /\left[z^{2}+\left(x-\eta_{0}\right)^{2}\right]^{1 / 2},
$$

and from (6.9) we get:

$$
f^{\prime}\left(\eta_{0}\right) / f\left(\eta_{0}\right)+i k_{1}\left(x-\eta_{0}\right) /\left[z^{2}+\left(x-\eta_{0}\right)^{2}\right]^{1 / 2}-i k_{1} \eta_{0} /\left(h^{2}+\eta_{0}^{2}\right)^{1 / 2}=0 .
$$

Hence

$$
g_{1}\left(\eta_{0}, \zeta_{0}, \tau_{0}\right)=\log f\left(\eta_{0}\right)-i k_{1}\left(h^{2}+\eta_{0}^{2}\right)^{1 / 2}-i k_{1}\left[z^{2}+\left(x-\eta_{0}\right)^{2}\right]^{1 / 2},
$$

where $\eta_{0}$ is given by (6.14).

We notice that $g_{1}\left(\eta_{0}, \zeta_{0}, \tau_{0}\right)$ is stationary when $\eta_{0}$ satisfies (6.14). Hence it does correspond to some stationary time path. Moreover if $\eta_{0}$ is the $x$-coordinate of a point on the boundary whose depth is small compared to $h$ and $z$, then the last two terms of (6.15) give the phase of the $P P$ wave reflected at that point.

It should be possible to identify equation (6.14) as the approximate condition for Snell's law to hold for the reflection. So we set up the exact Snell's condition for an incident irrotational wave and compare it to equation (6.14).

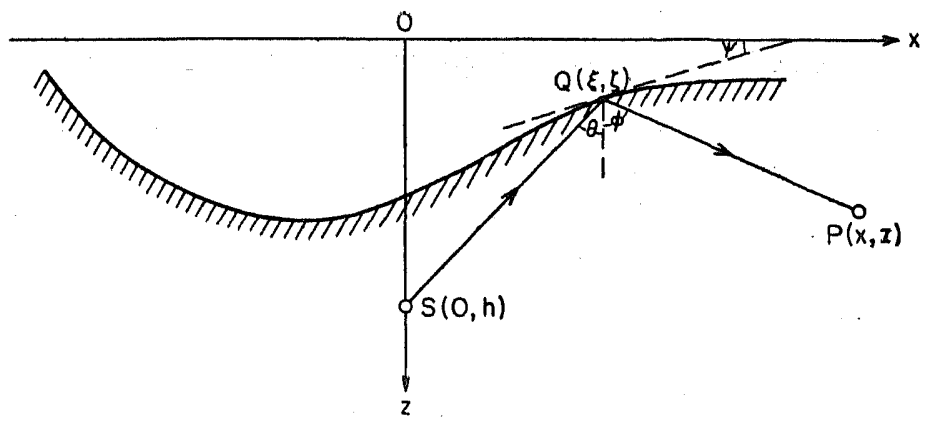

Fig. 1. An incident pulse $S Q$ and a reflected pulse $Q P$, making angles $\theta$ and $\varphi$ respectively with the vertical.

The condition for Snell's law to hold at $Q$ if the reflected ray $Q P$ is compressional is

where

$$
\phi-0=2 \phi \text {, }
$$

$$
\begin{aligned}
& \tan \theta=\xi /(h-\zeta), \\
& \tan \phi=(x-\xi) /(z-\zeta), \\
& \tan \phi=-(d z / d x)_{(\xi, \zeta)=-\varepsilon f^{\prime}(\xi) .}
\end{aligned}
$$

Hence substituting these values of $\theta, \phi, \phi$ into equation (6.16) we obtain on neglecting terms of $O\left(\varepsilon^{2}\right)$ :

$$
-2 \varepsilon f^{\prime}(\xi)=\frac{(x-\xi)(h-\zeta)-\xi(z-\zeta)}{(z-\zeta)(h-\zeta)+\xi(x-\zeta)}
$$

If in Fig. 1 we take $Q P$ as a reflected $S V$ ray, then the condition for Snell's law to hold at $Q$ is

$$
k_{1} \sin (\theta+\phi)=k_{2} \sin (\phi-\psi),
$$


where $k_{1}$ and $k_{2}$ are the wave numbers of the incident $P$ and the reflected $S V$ respectively. Using the expressions (6.17) equation (6.19) becomes:

$$
\begin{aligned}
-\varepsilon f^{\prime}(\xi)= & \left\{k_{2}(x-\xi)\left[(h-\zeta)^{2}+\xi^{2}\right]^{1 / 2}-k_{1} \xi\left[(z-\zeta)^{2}+(x-\xi)^{2}\right]^{1 / 2}\right\} \\
& /\left\{k_{1}(h-\zeta)\left[(z-\zeta)^{2}+(x-\xi)^{2}\right]^{1 / 2}+k_{2}(z-\zeta)\left[(h-\zeta)^{2}+\xi^{2}\right]^{1 / 2}\right\} .
\end{aligned}
$$

The saddle point of the integral (6.2) is given by

$$
\frac{\partial g_{2}}{\partial \eta}=\frac{\partial g_{2}}{\partial \zeta}=\frac{\partial g_{2}}{\partial \tau}=0
$$

From these equations we obtain the saddle point $\left(\eta_{0}, \zeta_{0}, \tau_{0}\right)$ of the integral $(6.2)$ as

$$
\left.\begin{array}{l}
\tau_{0}=k_{1} \eta_{0} /\left(h^{2}+\eta_{0}^{2}\right)^{1 / 2}, \\
\zeta_{0}=k_{2}\left(x-\eta_{0}\right) /\left[z^{2}+\left(x-\eta_{0}\right)^{2}\right]^{1 / 2},
\end{array}\right\}
$$

and $\eta_{0}$ is given by

$$
f^{\prime}\left(\eta_{0}\right) / f\left(\eta_{0}\right)+i k_{2}\left(x-\eta_{0}\right) /\left[z^{2}+\left(x-\eta_{0}\right)^{2}\right]^{1 / 2}-i k_{1} \eta_{0} /\left(h^{2}+\eta_{0}^{2}\right)^{1 / 2}=0,
$$

and from (6.4) we get

$$
g_{2}\left(\eta_{0}, \zeta_{0}, \tau_{0}\right)=\log f\left(\eta_{0}\right)-i k_{1}\left(h^{2}+\eta_{0}^{2}\right)^{1 / 2}-i k_{2}\left[z^{2}+\left(x-\eta_{0}\right)^{2}\right]^{1 / 2} .
$$

Here again we see that $g_{2}\left(\eta_{0}, \zeta_{0}, \tau_{0}\right)$ gives the phase of PS where the reflection occurs at the point $\left(\eta_{0}, \varepsilon\right)$ of the boundary where $\varepsilon / h$ and $\varepsilon / z$ are negligible.

In order to compare (6.14) with (6.18) and (6.20) with (6.22) we have to specify the function $f(x)$ and consider certain limiting cases.

\section{§7. Curvilinear Boundary}

Let

$$
f(x)=\exp \left(-a^{2} x^{2}\right) .
$$

The halfwidth of this curve is $2(\log 2)^{1 / 2} / a$. Then substituting this into (6.14) and (6.18) we get respectively (dropping the suffix zero on $\eta_{0}$ ):

$$
\begin{gathered}
-2 a^{2} \eta+i k_{1}(x-\eta) /\left[z^{2}+(x-\eta)^{2}\right]^{1 / 2}-i k_{1} \eta /\left(h^{2}+\eta^{2}\right)^{1 / 2}=0, \\
4 a^{2} \varepsilon \xi e^{-a^{2} \xi^{2}}=\frac{(x-\xi)(h-\zeta)-\xi(z-\zeta)}{(z-\zeta)(h-\zeta)+\xi(x-\xi)} .
\end{gathered}
$$

Let us consider two limiting cases.

(1) $a \ll 1, a \ll k_{1}$ and $z \ll x$.

Then neglecting the first term of (7.2) we obtain

$$
\eta=h x /(z+h)=\eta_{0}, \quad \text { say. }
$$

To obtain the second approximation we put $\eta=\eta_{0}+\delta_{1}$ in (7.2) which becomes:

$$
-2 a^{2}\left(\eta_{0}+\delta_{1}\right)+i k_{1}\left(x-\eta_{0}-\delta_{1}\right) /\left[z^{2}+\left(x-\eta_{0}-\delta_{1}\right)^{2}\right]^{1 / 2}-i k_{1}\left(\eta_{0}+\delta_{1}\right) /\left[h^{2}+\left(\eta_{0}+\delta_{1}\right)^{2}\right]^{1 / 2}=0 .
$$

On neglecting $\left(\delta_{1}^{2} / \eta_{0}^{2}\right)$ we obtain:

$$
\delta_{1} \doteqdot-2 a^{2} h \tan \psi /\left(2 a^{2}+\frac{i k_{1} R}{h z} \cos ^{4} \phi\right)
$$


where we have put

$$
x=R \sin \phi, \quad h+z=R \cos \phi .
$$

From (7.4) and (7.5) we have

$$
\left|\delta_{1} / \eta_{0}\right|=\left|2 a^{2} /\left(2 a^{2}+i k_{1} R \cos ^{4} \phi / h z\right)\right| .
$$

This is small as long as $a / k_{1}, h / k_{1}$ and $z / x$ are small. Hence under these assumptions $\eta=\eta_{0}$ gives a good approximation.

Putting the values of $\eta, \zeta$ and $\tau$ at the saddle point the index of the exponential (6.3) becomes:

$$
\begin{aligned}
g_{10}^{(1)} & \doteqdot g_{1}\left(\eta_{0}+\delta_{1}, \zeta_{0}, \tau_{0}\right) \\
& \doteqdot-i k_{1}\left[x^{2}+(z+h)^{2}\right]^{1 / 2}-a^{2} h^{2} \tan ^{2} \psi+O\left(a^{4}\right) .
\end{aligned}
$$

The first term of (7.8) is the phase of a $P$ wave which appears to come from the point $(0,-h)$ which is the image point of the source in the plane $z=0$.

From Snell's equation (7.3) we obtain the first approximation by neglecting the L.H.S.

$$
\xi=h x /(z+h) \equiv \xi_{0}, \quad \text { say . }
$$

This is the same as $\eta_{0},(7.4)$. To get the second approximation we put $\xi=\xi_{0}+\delta_{2}$ into (7.3) and we obtain after some manipulation

$$
\delta_{2} / \varepsilon \div \frac{2 \xi_{0}-x-a^{2} \xi_{0}\left(4 z h-3 x \xi_{0}+2 \xi_{0}^{2}\right)^{\prime}}{z+h-2 \varepsilon+2 a^{2} \varepsilon\left(2 z h+3 x \xi_{0}-3 \xi_{0}^{2}\right)} \div \frac{x(h-z)}{(h+z)^{2}} .
$$

Hence $\delta_{2}$ is $O(\varepsilon)$ which is negligible in the phase factor.

The length of the path traversed by the wave is

$$
\begin{aligned}
|S Q|+|Q P| & \doteqdot\left(h^{2}+\xi_{0}^{2}\right)^{1 / 2}+\left[\left(x-\xi_{0}\right)^{2}+z^{2}\right]^{1 / 2} \\
& =\left[x^{2}+(z+h)^{2}\right]^{1 / 2} .
\end{aligned}
$$

Hence the phase of the wave is $k_{1}\left[x^{2}+(z+h)^{2}\right]^{1 / 2}$. Thus the first approximation gives the same phase by the saddle point method as that derived by geometric ray theory.

(2) $a \gg 1, a \gg k_{1}$ and $x$ and $z$ are large.

From (7.2) the first approximation is

$$
\eta=0=\eta_{0}, \quad \text { say. }
$$

If for the second approximation we put $\eta=\eta_{0}+\delta_{1}$, we obtain from (7.2)

$$
\begin{aligned}
\delta_{1} & \doteq\left(i k_{1} \sin \psi\right) /\left[2 a^{2}+i k_{1}\left(R / h+\cos ^{2} \phi\right) / R\right] \\
& \doteqdot i k_{1} \sin \psi / 2 a^{2},
\end{aligned}
$$

where $z=R \cos \psi$ and $x=R \sin \psi$. Hence if $R$ and $a / k_{1}$ are both large then the first approximation $\eta=0$ is satisfactory.

Putting $\eta_{0}=\delta_{1}$ in (6.4) we get

$$
\begin{aligned}
g_{10}(2) & \doteqdot g_{1}\left(\delta_{1}, \zeta_{0}, \tau_{0}\right) \\
& \doteqdot-i k_{1}(h+R)-k_{1}^{2} \sin ^{2} \psi / 4 a^{2} .
\end{aligned}
$$

The corresponding first approximation of Snell's equation (7.3) is $\xi=0$. For the second approximation we get 


$$
\delta_{2} \doteqdot \frac{x(h-\varepsilon)}{z+h+4 a^{2} \varepsilon z h-2 \varepsilon}
$$

On neglecting $\zeta / z$ and $\delta_{2} / z$ and using the first approximation $\xi=0$, we have $|S Q|+|Q P|$ $=h+R$. Hence the phase of the wave is $k_{1}(h+R)$ which gives the same wave as (7.13).

(7.13) is the phase of a compressional wave which appears to have travelled from the source and is reflected at the origin to pass through the point of observation $(x, z)$. When $a$ is large the depression is a small one around the origin and so at distant points waves reflected at it appear to come from the origin.

However if we use the second approximation in determining the phase we obtain the same phase as (7.11).

We can obtain similar approximations for the reflected $S V$ wave. For the type of boundary (7.1), the saddle point and the Snell's equations (6.22) and (6.20) respectively become:

$$
\begin{aligned}
-2 a^{2} \eta+i k_{2}(x-\eta) /\left[z^{2}+(x-\eta)^{2}\right]^{1 / 2}-i k_{1} \eta /\left(h^{2}+\eta^{2}\right)^{1 / 2} 0, & \\
-2 \varepsilon \xi a^{2} e^{-a^{2} \xi^{2}=} & \left\{k_{2}(x-\xi)\left[(h-\zeta)^{2}+\xi^{2}\right]^{1 / 2}-k_{1} \xi\left[(z-\zeta)^{2}+(x-\xi)^{2}\right]^{1 / 2}\right\} \\
& \mid\left\{k_{1}(h-\zeta)\left[(z-\zeta)^{2}+(x-\xi)^{2}\right]^{1 / 2}+k_{2}(z-\zeta)\left[(h-\zeta)^{2}+\xi^{2}\right]^{1 / 2}\right\} .
\end{aligned}
$$

(1) $a \ll 1, a \leqslant k_{1}$ and $x \gg z$.

On this assumption we obtain from (7.15) as the first approximation, $y_{j a}$, which is given by the equation

$$
k_{2}\left(x-\eta_{0}\right) /\left[z^{2}+\left(x-\eta_{0}\right)^{2}\right]^{1 / 2}=k_{1} \eta_{0} /\left(h^{2}+\eta_{0^{2}}\right)^{1 / 2}
$$

and the second approximation yields a correction term

$$
\delta_{1} \div-2 a^{2} \eta_{0} /\left[2 a^{2}+i k_{2}\left(z^{2} / R_{2}^{3}\right)+i k_{1}\left(h^{2} / R_{1}^{3}\right)\right]^{1 / 2},
$$

where $R_{1}=\left(h^{2}+\eta_{0}^{2}\right)^{1 / 2}$ and $R_{2}=\left[z^{2}+\left(x-\gamma_{0}\right)^{2}\right]^{1 / 2},\left|\partial_{1} / \gamma_{0}\right|<1$ if $a / k_{1}$ is small since $R_{z}$ is small.

Since $\varepsilon$ is small we neglect the L.H.S. of $(7.16)$ and if in the R. H.S. we neglect $\zeta / z$ we obtain the first approximation as $\xi_{0}$, given by

$$
k_{2}\left(x-\xi_{0}\right) /\left[z^{2}+\left(x-\xi_{0}\right)^{2}\right]^{1 / 2}=k_{1} \xi_{0} /\left(h^{2}+\xi_{0}^{2}\right)^{1 / 2} .
$$

Comparing equations (7.17) and (7.19) we see that $\hat{5}_{0} \cdots \eta_{i 0} .(7.17)$ (or $(7.19)$ can be recognized as the equation for Snell's law of reflection when an incident $P$ wave generates a reflected $S V$ wave at a plane bounclary at the point $\left(y_{0}, 0\right)$.

The phase of such a wave is given by $(6.23)$ and it is seen to be a $l 5$.

(2) $a \gg 1, a \gg k_{1}$; and $x$ and $z$ are large; $k_{1}>h$.

We obtain the first approximations as $\eta \ldots \ldots=0$, and the correction term for $\eta$ is

$$
\begin{aligned}
\delta_{4} & \doteqdot i k_{2} \sin \phi /\left[2 a^{2}+i\left(R k_{1} / h+k_{2} \cos ^{2} \phi\right) / R\right] \\
& \doteqdot i k_{2} \sin \phi / 2 a^{2},
\end{aligned}
$$

where $x=R \sin \phi, z=R \cos \phi$. Hence the first approximation is good if $k_{1} / h$ and $a / k_{2}$ are large.

Putting $\eta_{0}=\delta_{4}$ into $(6.23)$ we obtain the exponent as

$$
g_{20}{ }^{(2)} \div-i k_{1} h-i k_{2} R-k_{2}^{2} \sin ^{2} y_{1} 4 a^{2}
$$

This is the phase of a PS which travelled from the source to the origin as $P$ and is reflected there as $S V$ wave.

The Hessian of $g_{1}$ and $g_{2}$ at the saddle point $\left(\gamma_{10}, \zeta_{0}, r_{0}\right)$ are respectively 


$$
\left.\begin{array}{l}
\Delta_{1}=k_{1}^{2}\left(z \bar{v}_{1}^{3}+h v_{1}^{3}-2 a^{2} z h k_{1}^{2}\right) / \bar{v}_{1}^{3} v_{2}^{3}, \\
\Delta_{2}=\left(z k_{2}^{2} \bar{v}_{1}^{3}+h k_{1}^{2} v_{2}^{3}-2 a^{2} z h k_{1}^{2} k_{2}^{2}\right) / \bar{v}_{1}^{3} v_{2}^{3},
\end{array}\right\}
$$

where for $\Delta_{1}, \zeta_{0}, \tau_{0}$ and $\eta_{0}$ are given by (6.13) and (6.14) and for $\Delta_{2}$ their values are given by (6.21) and (6.22).

Hence applying the formula (6.12) we obtain the steepest descent approximation for the integrals (6.1) and (6.2).

(i) If $a \ll 1, x \gg z$, and $a \ll k_{1}$ and $k_{1} / h \gg 1$ and if we write

$$
x=R_{1} \sin \psi, \quad z+h=R_{1} \cos \psi,
$$

we obtain by using the approximation (7.4)

$$
\phi_{p 1}=\frac{\varepsilon(-2 \pi)^{3 / 2}}{\pi A_{1} \Delta_{p 1}{ }^{1 / 2}} G_{p 1} \exp \left\{-i k_{1}\left[x^{2}+(z+h)^{2}\right]^{1 / 2}-a^{2} h^{2} \tan ^{2} \phi\right\},
$$

where

$$
\begin{aligned}
& G_{p 1}=\left\{\left(k_{2}{ }^{2}-2 k_{1}{ }^{2} \sin ^{2} \psi\right)^{4}-16 k_{1}{ }^{3} \sin ^{3} \psi\left(k_{2}{ }^{2}-k_{1}{ }^{2} \sin ^{2} \psi\right)\right. \\
& \left.\times\left[k_{1} \sin \psi\left(k_{2}{ }^{2}-k_{1}{ }^{2} \sin ^{2} \psi\right)^{1 / 2}-2 a^{2} h \tan \psi\left(k_{2}{ }^{2}-k_{1}{ }^{2}\right)\right]\right\}, \\
& \Delta_{p 1}=R_{1}\left(2 a^{2} h+i k_{1} \cos ^{3} \phi\right) / k_{1}^{2} \cos ^{5} \phi \text {, } \\
& \left.A_{1}=\left[\left(k_{2}{ }^{2}-2 k_{1}{ }^{2} \sin ^{2} \phi\right)^{2}+4 k_{1}{ }^{3} \sin ^{2} \phi \cos \phi\left(k_{2}{ }^{2}-k_{1}{ }^{2} \sin ^{2} \phi\right)^{1 / 2}\right]^{2} .\right)
\end{aligned}
$$

(7.24) is the potential of a $P$ wave which appears to come from the image point, $(0,-h)$, of the source. Its amplitude is proportional to $R_{1}^{-1 / 2}$ or (distance) $)^{-1 / 2}$. $\psi$ is the angle of reflection of the wave.

From the equation (7.17) it is obvious that $\eta_{0}$ must have the dimension of length. Thus we can write

$$
z=R \cos \phi, \quad x-\eta_{0}=R \sin \phi, \quad h=R_{3} \cos \theta, \quad \eta_{0}=R_{3} \sin \theta .
$$

Therefore the relation (7.17) can be written as

$$
k_{2} \sin \phi=k_{1} \sin \theta .
$$

This is Snell's law when a $P(S V)$ wave incident on a plane boundary at an angle $\theta(\phi)$ is partially reflected as an $S V(P)$ wave with an angle of reflection $\phi(\theta)$.

Using the approximations obtained, we get the value of the integral (6.2) as

$$
\phi_{s 1}=\frac{\varepsilon(-2 \pi)^{3 / 2} G_{2}}{\pi B_{1} B_{2} \Delta_{s 1} 1^{1 / 2}} \exp \left[-i k_{1} R_{3}-i k_{2} R-a^{2} R_{3}^{2} \sin ^{2} \theta\right],
$$

where

$$
\begin{aligned}
& B_{1}=\left(k_{2}^{2}-2 k_{1}^{2} \sin ^{2} \theta\right)^{2}+4 k_{2}{ }^{2} k_{1} \sin ^{2} \theta \cos \theta\left(k_{2}{ }^{2}-k_{1}^{2} \sin ^{2} \theta\right)^{1 / 2}, \\
& B_{2}=k_{2}{ }^{4} \cos ^{2} \phi+4 k_{2}{ }^{3} \sin ^{2} \phi \cos \phi\left(k_{1}{ }^{2}-k_{2}{ }^{2} \sin ^{2} \phi\right)^{1 / 2} \text {, } \\
& G_{2}=2 k_{2} \sin \phi\left(k_{2}^{2}-2 k_{1}^{2} \sin ^{2} \theta\right)\left(k_{1}^{2}-k_{2}^{2} \sin ^{2} \phi\right)^{1 / 2} \\
& \times\left[\left(k_{2}{ }^{2}-2 k_{1}{ }^{2} \sin ^{2} \theta\right)^{2}+4 k_{1}{ }^{2} \sin ^{2} \theta\left(k_{2}{ }^{2}-k_{1}{ }^{2} \sin ^{2} \theta\right)^{1 / 2}\right] \\
& +2 k_{2}{ }^{2} \cos 2 \phi\left\{k_{1} \sin \theta\left(k_{2}{ }^{2}-k_{1}{ }^{2} \sin ^{2} \theta\right)^{1 / 2}\right. \\
& \times\left[\left(k_{2}{ }^{2}-2 k_{1}{ }^{2} \sin ^{2} \theta\right)^{2}+k_{1}{ }^{4} \sin ^{2} 2 \theta\right] \\
& \left.+i 8 a^{2} k_{1}^{2} \sin ^{3} \theta R_{3}\left(k_{2}^{2}-k_{1}^{2} \sin ^{2} \theta\right)^{1 / 2}\left(k_{2}^{2}-k_{1}^{2}\right)\right\} \text {, } \\
& \Delta_{s 1}=\left(i R k_{1} \cos ^{3} \theta+i h k_{2} \cos ^{2} \phi+2 a^{2} R h\right) / k_{1} k_{2} \cos ^{2} \phi \cos ^{3} \theta \text {. }
\end{aligned}
$$


Thus (7.28) represents a wave which traversed the distance $R_{8}$, from the source, as a $P$ and then was converted at the boundary into an $S V$ and traverses the distance $R$ to the point of observation. This is the $P S$. The coordinates of the point of reflection is $\left(\eta_{0}, 0\right)$.

(ii) If $a, a / k_{1}$ and $k_{1} / h_{1}$ and $k_{1} / h$ are large, and if we write

$$
x=R_{2} \sin \psi, \quad z=R_{2} \cos \psi,
$$

we obtain by using the approximation (7.12)

where

$$
\phi_{p 2} \doteqdot \frac{\varepsilon(-2 \pi)^{3 / 2} k_{2}^{2}\left(k_{2}^{2}-2 k_{1}^{2} \sin ^{2} \psi\right)}{\pi A_{2} \Delta_{p 2}{ }^{1 / 2}} \exp \left[-i k_{1}\left(h+R_{2}\right)-k_{1}^{2} \sin ^{2} \phi / 4 a^{2}\right]
$$

$$
\left.\begin{array}{l}
A_{2}=\left(k_{2}^{2}-2 k_{1}^{2} \sin ^{2} \phi\right)^{2}+4 k_{1}^{3} \sin ^{2} \phi \cos \phi\left(k_{2}^{2}-k_{1}^{2} \sin ^{2} \phi\right)^{1 / 2} \\
\Delta_{p 2}=R_{2}\left(i k_{1}+i k_{1} h \cos ^{2} \phi+2 a^{2} h\right) / k_{1}^{2} \cos ^{2} \psi
\end{array}\right\}
$$

(7.31) is the potential of a wave which travelled as $P$ from the source located at $(0,+h)$ and appeared to be reflected as a $P$ wave from the origin of coordinates. Its amplitude is proportional to (distance) $)^{-1 / 2}$.

Similarly for the second integral (6.2) using the approximation (7.20) we obtain

$$
\phi_{82}=\frac{2 \varepsilon(-2 \pi)^{3 / 2} \sin \psi\left(k_{1}^{2}-k_{2}^{2} \sin ^{2} \psi\right)^{1 / 2}}{\pi B_{8} A_{82}{ }^{1 / 2}} \exp \left[-i k_{1} h-i k_{2} R_{2}-k_{2}{ }^{2} \sin ^{2} \psi / 4 a^{2}\right],
$$

where

$$
\left.\begin{array}{l}
B_{3}=k_{2} \cos ^{2} 2 \psi+4 \sin ^{2} \phi \cos \phi\left(k_{1}^{2}-k_{2}{ }^{2} \sin ^{2} \psi\right)^{1 / 2}, \\
\Delta_{s 2}=\left(i k_{1} R_{2}+2 a^{2} h R_{2}+i h k_{2} \cos ^{2} \phi\right) / k_{1} k_{2} \cos ^{2} \psi
\end{array}\right\}
$$

(7:33) is the potential of a wave travelling from the point source to the epicentre as a compressional wave and then is reflected as a shear wave.

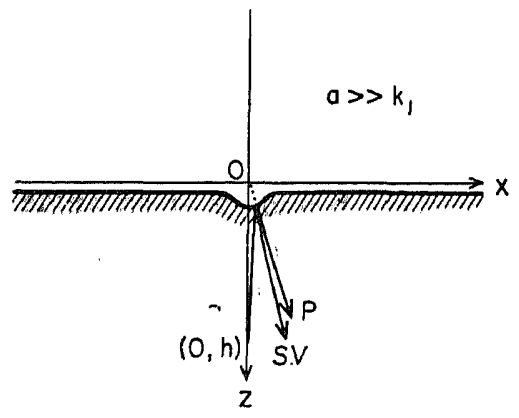

Fig. 2. The secondary waves appear to diverge from the centre of symmetry, $O$, of the basin when $a \leqslant k_{1}$ and $a \gg 1$.

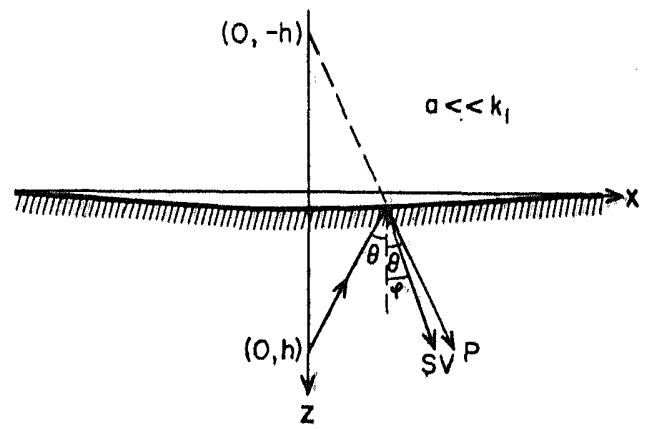

Fig. 3. The secondary waves are reflected from. the surface as though it were flat for $a \ll k_{1}$ and $a \leqslant 1$.

\section{§. Sinusoidal Boundary}

Let

$$
\varepsilon f(x)=\varepsilon(1-\cos n p x) \text {. }
$$

Then the saddle point equation (6.14) and Snell's condition (6.18) become, respectively

$$
\frac{n p \sin (n p \eta)}{-\cos n p \eta}+i k_{1}(x-\eta) /\left[z^{2}+(x-\eta)^{2}\right]^{1 / 2}-i k_{1} \eta /\left(h^{2}+\eta^{2}\right)^{1 / 2}=0,
$$




$$
-2 \varepsilon n p \sin (n p \xi)=\frac{(x-\xi)(h-\zeta)-\xi(z-\zeta)}{(z-\zeta)(h-\zeta)+\xi(x-\xi)}
$$

Here again in order to compare the two equations (8.2) and (8.3) we carry out similar approximations as in $(\S 7)$. We give only a brief account of it.

(i) If $n p / k_{1}, n p$ and $x /(h+z)$ are small, then from (8.2) the first approximation is (by neglecting the first term of the equation)

$$
\eta=x h /(x+h)=\eta_{0}, \quad \text { say. }
$$

Putting $\eta=\eta_{0}+\delta_{1}$ in (8.2) we obtain the correction term as

$$
\delta_{1} \doteqdot \frac{-i\left(n p / k_{1}\right) \sin \left(n p \eta_{0}\right)}{\left(1-\cos n p \eta_{0}\right)(z+h)^{4} / z h R^{3}}
$$

where

$$
R=\left[x^{2}+(z+h)^{2}\right]^{1 / 2} .
$$

Hence if $n p / k_{1} \ll 1$ the correction term $\delta_{1}$ is negligible compared with the first approximation $\eta_{0}$.

By putting $\eta=\eta_{0}+\delta_{1}$ in (6.3) we obtain

$$
\begin{aligned}
g_{10}^{(1)} & \doteqdot \log f\left(\eta_{0}+\delta_{1}\right)-i k_{1}\left[h^{2}+\left(\eta_{0}+\delta_{1}\right)^{2}\right]^{1 / 2}-i k_{1}\left[z^{2}+\left(x-\eta_{0}-\delta_{1}\right)^{2}\right]^{1 / 2} \\
& =\log \left[1-\cos n p\left(\eta_{0}+\delta_{1}\right)\right]-i k_{1} R .
\end{aligned}
$$

Thus this approximation shows that the reflected $P$ wave appears to come from the image of the source in the plane $z=0$.

For the first approximation of (8.3) we get $\xi=x h /(z+h)=\xi_{0}$, say. This agrees with (8.4). For the second approximation we put $\xi=\xi_{0}+\delta_{2}$ and by neglecting $\zeta / z$ in the R. H. S. we obtain

$$
\delta_{2} \doteqdot \frac{-2 \varepsilon n p \sin \left(n p \eta_{0}\right)}{(h+z)^{3} / h z R^{2}}=\frac{-2 \varepsilon n p \sin \left(n p \eta_{0}\right)}{(h+z)^{4} / h z R^{3}}
$$

since $x /(z+h)$ is small.

(ii) $n p / k_{1}$ and $n p$ are large.

The first approximation of (8.2) is given by (neglecting the last two terms of the equation)

$$
\frac{n p \sin n p \eta}{1-\cos n p \eta}=0
$$

i. e. $n p \eta=s \pi$, where $s$ is an integer, positive or negative. But since if $s$ is even $1-\cos n p \eta$ $=0$, we take only odd ones. Hence

$$
\eta=(2 m-1) \pi / n p=\eta_{0}, \quad \text { say. }
$$

Putting $\eta=\eta_{0}+\delta_{3}$ in (8.2) and assuming that $\sin \left(n p \delta_{3}\right) \doteqdot n p \delta_{3}$, we obtain the correction term as

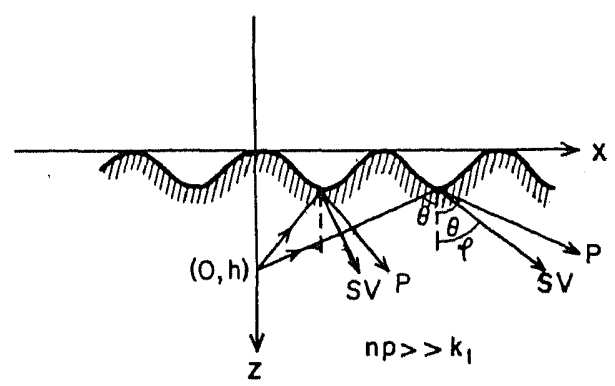

Fig. 4. The secondary waves are reflected only from the troughs of the sinusoidal surface for $n p \gg k_{1}$ and $n p \gg 1$.

$$
\delta_{3} \doteqdot \frac{2 i k_{1}}{n^{2} p^{2}}\left\{\left(x-\eta_{0}\right) /\left[z^{2}+\left(x-\eta_{0}\right)^{2}\right]^{1 / 2}-\eta_{0} /\left(h^{2}+\eta_{0}{ }^{2}\right)^{1 / 2}\right\} \text {. }
$$

Hence if $n p\left|k_{1} \gg 1,\right| \delta_{3} / \eta_{0} \mid \leqslant 1$ and therefore the first approximation is good. If we assume that $z \gg\left(x-\eta_{0}\right)$ and $h \gg \eta_{0}$, then by expanding the radicals in (8.9) into series we obtain 


$$
\delta_{3}{ }^{\prime}=2 i k_{1}\left[x h-\eta_{0}(h+z)\right] / n^{2} p^{2} h z .
$$

The index of the exponential (6.3) is

$$
\begin{aligned}
g_{10}{ }^{(2)} \doteqdot & \log f\left(\eta_{0}+\delta_{3}\right)-i k_{1}\left[h^{2}+\left(\eta_{0}+\delta_{3}\right)^{2}\right]^{1 / 2}-i k_{1}\left[z^{2}+\left(x-\eta_{0}-\delta_{8}\right)^{2}\right]^{1 / 2} \\
= & \log \left[1-\cos n p\left(\eta_{0}+\delta_{3}\right)\right] \\
& -\frac{2 k_{1}^{2}}{n^{2} p^{2}}\left\{\left(x-\eta_{0}\right) /\left[z^{2}+\left(x-\eta_{0}\right)^{2}\right]^{1 / 2}-\eta_{0} /\left(h^{2}+\eta_{0}^{2}\right)^{1 / 2}\right\} \\
& -i k_{1}\left[h^{2}+(2 m-1)^{2} \pi^{2} / n^{2} p^{2}\right]^{1 / 2}-i k_{1}\left\{z^{2}+[x-\pi(2 m-1) / n p]^{2}\right\}^{1 / 2} .
\end{aligned}
$$

This gives the phase of the $P P$ waves reflected from the troughs if the depths of these troughs are considered negligible compared to $h$ and $z$. This is the case when the wavelength of the incident waves is large compared to that of the surface. Thus the surface behaves as a grating.

As a first approximation of Snell's equation (8.3) we get

i. e.

$$
\sin n p \xi=0 \text {, }
$$

where $m$ is any positive or negative integer. $\xi_{0}$ is the coordinate of a trough or a peak according as $m$ is odd or even.

Inserting $\xi=\xi_{0}+\delta_{4}$ in (8.3) we obtain after some reduction

$$
\delta_{4} \doteqdot \pm\left[h x-\xi_{0}(h+z)\right] / 2 \varepsilon n^{2} p^{2} h z
$$

where \pm is taken according as $m$ is even or odd.

$$
\left|\frac{\delta_{4}}{\xi_{0}}\right| \leqslant 1 \quad \text { if } \quad|n p| \geqslant 1
$$

The values of the Hessians of $g_{1}$ and $g_{2}$ at the saddle point $\left(\eta_{0}, \zeta_{0}, \tau_{0}\right)$ are respectively

$$
\left.\begin{array}{rl}
\Delta_{1}= & k_{1}{ }^{2}\left[z \bar{v}_{1}{ }^{3}+h v_{1}^{3}+z h k_{1}^{2} n^{2} p^{2} \cos n p \eta /(1-\cos n p \eta)\right. \\
& \left.-z h k_{1}^{2} n p \sin n p \eta /(1-\cos n p \eta)^{2}\right] / v_{1}^{3} \bar{v}_{1}^{3}, \\
\Delta_{2}= & {\left[z k_{2}{ }^{2} \bar{v}_{1}^{3}+h k_{1}^{2} v_{2}^{3}+z h k_{1}{ }^{2} k_{2}{ }^{2} n^{2} p^{2} \cos n p \eta /(1-\cos n p \eta)\right.} \\
& \left.-z h k_{1}{ }^{2} k_{2}{ }^{2} n p \sin n p \eta /(1-\cos n p \eta)^{2}\right] / \bar{v}_{1}^{3} v_{2}{ }^{3} .
\end{array}\right\}
$$

Using the saddle point approximation formula (6.12) we can work out the approximate amplitude of the reflected waves.

Hence if $n p / k_{1} \ll 1, n p \ll 1$ and $x /(h+z) \ll 1$, writing

$$
x=R_{1} \sin \psi, \quad z+h=R_{1} \cos \phi,
$$

we obtain by using the approximation (8.4)

where

$$
\phi_{p 1} \doteqdot \frac{\varepsilon(-2 \pi)^{3 / 2} G_{p 1}}{\pi A A_{p 1}{ }^{1 / 2}} \exp \left\{-i k_{1}\left[x^{2}+(z+h)^{2}\right]^{1 / 2}-\log [1-\cos n p(h \tan \psi)]\right\}
$$

$$
\begin{aligned}
G_{p 1}= & \left(k_{2}^{2}-2 k_{1}{ }^{2} \sin ^{2} \phi\right)^{4}-16 k_{1}{ }^{3} \sin ^{3} \psi\left(k_{2}{ }^{2}-k_{1}{ }^{2} \sin ^{2} \psi\right)^{3 / 2} \\
& \times\left[k_{1} \sin \psi\left(k_{2}{ }^{2}-k_{1}{ }^{2} \sin ^{2} \psi\right)+\left(k_{2}{ }^{2}-k_{1}{ }^{2}\right) \frac{n p \sin n p \eta_{0}}{1-\cos n p \eta_{0}}\right],
\end{aligned}
$$




$$
\begin{aligned}
& A=\left[\left(k_{2}^{2}-2 k_{1}{ }^{2} \sin ^{2} \phi\right)^{2}+4 k_{1}{ }^{3} \sin ^{2} \psi \cos \phi\left(k_{2}{ }^{2}-k_{1}{ }^{2} \sin ^{2} \psi\right)^{1 / 2}\right]^{2}, \\
& \Delta_{p 1}=\left[i k_{1}(z+h) \cos ^{3} \psi-\frac{z h n^{2} p^{2} \cos n p \eta_{0}}{1-\cos n p \eta_{0}}+\frac{z h n p \sin n p \eta_{0}}{\left(1-\cos n p \eta_{0}\right)^{2}}\right] / k_{1}{ }^{2} \cos ^{6} \psi
\end{aligned}
$$

(8.16) is the displacement potential of a $P$ wave which appears to come from the image point, $(0,-h)$ of the source.

If $n p / k_{1} \gg 1$ and $n p \gg 1$ then writing

$$
\eta_{0}=R_{1} \sin \phi, \quad h=R_{1} \cos \phi, \quad x-\eta_{0}=R_{2} \sin \theta \text { and } z=R_{2} \cos \theta,
$$

we obtain from the approximation (8.8)

$$
\phi_{p 2} \doteqdot \frac{\varepsilon(-2 \pi)^{3 / 2} G_{p 2}}{\pi A_{1} A_{2} \Delta_{p 2}{ }^{1 / 2}} \exp \left(g_{10}\right)
$$

where

$$
\begin{aligned}
& g_{10}=\log \left(1-\cos n p \eta_{0}\right)-\frac{2 k_{1}^{2}}{n^{2} p^{2}}(\sin \theta-\sin \phi)-i k_{1} R_{1}-i k_{2} R_{2}, \\
& G_{p 2}=\left(k_{2}{ }^{2}-2 k_{1}{ }^{2} \sin ^{2} \theta\right)\left(k_{2}{ }^{2}-2 k_{1}{ }^{2} \sin ^{2} \phi\right)\left[\left(k_{2}{ }^{2}-2 k_{1}{ }^{2} \sin ^{2} \phi\right)^{2}\right. \\
& \left.+4 k_{1}{ }^{2} \sin ^{2} \phi\left(k_{2}{ }^{2}-k_{1}{ }^{2} \sin ^{2} \phi\right)\right]+4 k_{1} \sin \theta\left(k_{2}{ }^{2}-k_{1}{ }^{2} \sin ^{2} \theta\right)^{1 / 2} \\
& \times\left\{-k_{1} \sin \phi\left(k_{2}{ }^{2}-k_{1}{ }^{2} \sin ^{2} \phi\right)^{1 / 2}\left[\left(k_{2}{ }^{2}-2 k_{1}{ }^{2} \sin ^{2} \phi\right)^{2}+k_{1}{ }^{2} \sin ^{2} 2 \phi\right]\right. \\
& \left.+4 i k_{1}^{2} \sin ^{2} \phi\left(k_{2}^{2}-k_{1}^{2}\right)\left(k_{2}^{2}-k_{1}^{2} \sin ^{2} \phi\right)^{1 / 2} \frac{n p \sin n p \eta_{0}}{1-\cos n p \eta_{0}}\right\}, \\
& A_{1}=\left(k_{2}{ }^{2}-2 k_{1}{ }^{2} \sin ^{2} \phi\right)^{2}+4 k_{1}{ }^{8} \sin ^{8} \phi \cos \phi\left(k_{2}^{2}-k_{1}^{2} \sin ^{2} \phi\right)^{1 / 2} \text {, } \\
& A_{2}=\left(k_{2}{ }^{2}-2 k_{1}{ }^{2} \sin ^{2} \theta\right)^{2}+4 k_{1}{ }^{3} \sin ^{3} \theta \cos \theta\left(k_{2}{ }^{2}-k_{1}{ }^{2} \sin ^{2} \theta\right)^{1 / 2} \text {, } \\
& \Delta_{p 2}=\left[i z k_{1} \cos ^{3} \phi+i h k_{1} \cos ^{3} \theta-\frac{z h n^{2} p^{2} \cos n p \eta_{0}}{1-\cos n p \eta_{0}}+\frac{z h n p \sin n p \eta_{0}}{\left(1-\cos n p \eta_{0}\right)^{2}}\right] / k_{1}^{2} \cos ^{8} \theta \cos ^{3} \phi \text {. }
\end{aligned}
$$

(8.19) is the potential of a wave which travelled from the source to one of the troughs as $P$ and reflected from there as a shear wave.

We can obtain similar approximations for the reflected $S V$ wave. For the sinusoidal boundary (8.1), the saddle point and the Snell's equation (6.22) and (6.20) respectively become:

$$
\begin{gathered}
\frac{n p \sin (n p \eta)}{1-\cos n p \eta}+\frac{i k_{2}(x-\eta) .}{\left[z^{2}+(x-\eta)^{2}\right]^{1 / 2}}-\frac{i k_{1} \eta}{\left(h^{2}+\eta^{2}\right)^{1 / 2}}=0, \\
\varepsilon n p \sin (n p \xi)+\left\{k_{2}(x-\xi)\left[(h-\zeta)^{2}+\xi^{2}\right]^{1 / 2}-k_{1}^{2} \xi\left[(z-\zeta)^{2}+(x-\zeta)^{2}\right]^{1 / 2}\right\} \\
/\left\{k_{1}(h-\zeta)\left[(z-\zeta)^{2}+(x-\zeta)^{2}\right]^{1 / 2}+k_{2}(z-\zeta)\left[(h-\zeta)^{2}+\xi^{2}\right]^{1 / 2}\right\}=0 .
\end{gathered}
$$

(i) If $n p / k_{1}$ and $n p$ are small then from (8.21) we get approximately,

$$
\frac{k_{2}(x-\eta)}{\left[z^{2}+(x-\eta)^{2}\right]^{1 / 2}}=\frac{k_{1} \eta}{\left(h^{2}+\eta^{2}\right)^{1 / 2}}
$$

Equation (8.23) gives the first approximation for $\eta=\eta_{0}$, say. To obtain the second approximation we put in (8.21) $\eta=\eta_{0}+\delta_{1}$ and on neglecting third order quantities, we obtain:

$$
\begin{aligned}
\delta_{1} \doteqdot & -i n p \sin \left(n p \eta_{0}\right) /\left[\left\{k_{2} z^{2} /\left[z^{2}+\left(x-\eta_{0}\right)^{2}\right]^{3 / 2}+k_{1} h^{2} /\left(h^{2}+\eta_{0}^{2}\right)^{3 / 2}\right\}\right. \\
& \left.\times\left(1-\cos n p \eta_{0}\right)+i n^{2} p^{2} \cos n p \eta_{0}\right] .
\end{aligned}
$$


Let us put

$$
\left.\begin{array}{rlrl}
z & =R_{2} \cos \phi, & x-\eta_{0} & =R_{2} \sin \phi . \\
h=R_{1} \cos \theta, \quad \eta_{0} & =R_{1} \sin \theta .
\end{array}\right\}
$$

Then expressions (8.22) and (8.24) become

$$
k_{2} \sin \phi=k_{1} \sin \theta \text {, }
$$

which is the well known Snell's law of reflection where $\theta$ and $\phi$ are the angles of reflection of the $P$ and $S V$ waves respectively.

$$
\delta_{1} \doteqdot \frac{i n p \sin n p \eta_{0}}{k_{2} \cos ^{2} \phi / R_{2}+k_{1} \cos ^{2} \theta / R_{1}} .
$$

Hence if $n p / k_{1} \ll 1$ then $\left|\delta_{1} / \eta_{0}\right|$ is small.

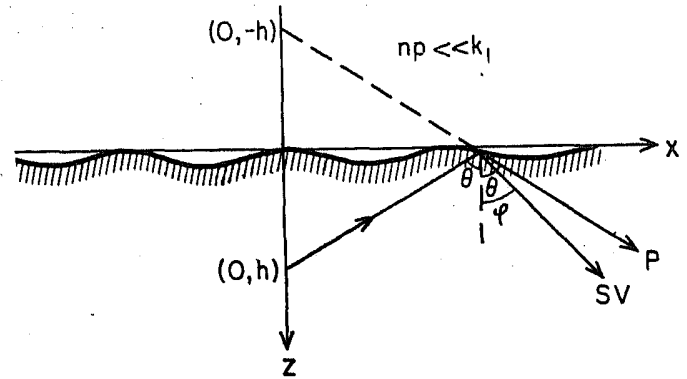

Fig. 5. The secondary waves are reflected as though the surface is specular in nature when $n p \ll k_{1}$ and $n p \ll 1$.

Since $n p / k_{1}$ and $n p$ are small for the first approximation we neglect the first term in equation (8.22) and neglecting $\zeta / z$ in the second term we obtain

$$
\frac{k_{2}\left(x-\xi_{0}\right)}{\left[z^{2}+\left(x-\xi_{0}\right)^{2}\right]^{1 / 2}}=\frac{k \xi_{0}}{\left(h^{2}+\xi_{0}^{2}\right)^{1 / 2}} .
$$

Comparing (8.28) and (8.23) we see that $\xi_{0}=\eta_{0}$.

The phase of the reflected wave is from (6.23) approximately

$$
g_{2}\left(\eta_{0}, \zeta_{0}, \tau_{0}\right)=\log \left(1-\cos n p \eta_{0}\right)-i k_{1} R_{1}-i k_{2} R_{2},
$$

where $\eta_{0}$ is given by equation (8.23).

The saddle point approximation formula (6.12) gives the potential of the wave as:

$$
\psi_{s 1}=\frac{\varepsilon(-2 \pi)^{3 / 2} G_{2}}{\pi B_{1} B_{2} \Delta_{81}{ }^{1 / 2}} \exp \left[-i k_{1} R_{1}-i k_{2} R_{2}+\log \varepsilon\left(1-\cos n p \eta_{0}\right)\right],
$$

where

$$
\begin{aligned}
& \left.\begin{array}{l}
B_{1}=\left(k_{2}^{2}-2 k_{1}^{2} \sin ^{2} \theta\right)^{2}+4 k_{1} k_{2}{ }^{2} \sin ^{2} \theta \cos \theta\left(k_{2}{ }^{2}-k_{1}{ }^{2} \sin ^{2} \theta\right)^{1 / 2}, \\
B_{2}=k_{2}{ }^{4} \cos 2 \phi+4 k_{2}{ }^{3} \sin ^{2} \phi \cos \phi\left(k_{1}{ }^{2}-k_{2}{ }^{2} \sin ^{2} \phi\right)^{1 / 2}
\end{array}\right\} \\
& G_{2}=2 k_{2} \sin \phi\left(k_{2}{ }^{2}-2 k_{1}{ }^{2} \sin ^{2} \theta\right)\left(k_{1}^{2}-k_{2}{ }^{2} \sin ^{2} \phi\right)^{1 / 2} \\
& \times\left[\left(k_{2}^{2}-2 k_{1}^{2} \sin ^{2} \theta\right)^{2}+4 k_{1}^{2} \sin ^{2} \theta\left(k_{2}^{2}-k_{1}^{2} \sin ^{2} \theta\right)^{1 / 2}\right] \\
& +2 k_{2}^{2} \cos 2 \phi\left\{k_{1} \sin \theta\left(k_{2}{ }^{2}-k_{1}^{2} \sin ^{2} \theta\right)^{1 / 2}\left[\left(k_{2}^{2}-2 k_{1}^{2} \sin ^{2} \theta\right)^{2}+k^{4} \sin ^{2} 2 \theta\right]\right. \\
& \left.+\frac{i 4 n p k_{1}^{2} \sin ^{2} \theta \sin \left(n p \eta_{0}\right)}{1-\cos n p \eta_{0}}\left(k_{2}^{2}-k_{1}^{2}\right)\left(k_{2}^{2}-k_{1}^{2} \sin ^{2} \theta\right)^{1 / 2}\right\} \\
& \Delta_{s 1}=\left[i z k_{1} \cos ^{3} \theta+i h k_{2} \cos ^{3} \phi-\frac{z h n^{2} p^{2} \cos n p \eta_{0}}{1-\cos n p \eta_{0}}+\frac{z h n p \sin \left(n p \eta_{0}\right)}{\left(1-\cos n p \eta_{0}\right)^{2}}\right] / k_{1} k_{2} \cos ^{3} \theta \cos ^{3} \phi .
\end{aligned}
$$

(8.30) represents a wave which traversed the distance $R_{1}$ from the source as compressional wave and then was converted at the boundary into a shear wave and traverses the distance $R_{2}$ to the point of observation. This is the PS.

(ii) If $n p / k_{1} \gg 1$ and $n p \gg 1$ then from (8.21) we get by neglecting the second term of the equation

$$
\frac{n p \sin n p \eta}{1-\cos n p \eta}=0
$$


i. e.

$$
\eta=(2 m-1) \pi / n p=\eta_{0}, \quad \text { say. }
$$

This is the first approximation. To obtain a correction terms to this approximation we put $\eta=\eta_{0}+\delta_{2}$ in (8.21).

$$
\delta_{2} \doteqdot\left[\frac{k_{2}(x-\eta)}{R_{2}}-\frac{k_{1} \eta}{R_{1}}\right] /\left[\frac{k_{2} z^{2}}{R_{2}{ }^{3}}+\frac{k_{1} h^{2}}{R_{1}^{3}}-i n^{2} p^{2}\right]
$$

where

$$
R_{2}=\left[z^{2}+\left(x-\eta_{0}\right)^{2}\right]^{1 / 2}, \quad R_{1}=\left(h^{2}+\eta^{2}\right)^{1 / 2} .
$$

If we put $x-\eta_{0}=R_{2} \sin \phi, \eta_{0}=R_{1} \sin \theta$ then (8.35) becomes

$$
\delta_{2} \doteqdot\left(k_{2} \sin \phi-k_{1} \sin \theta\right) /\left(\frac{k_{2} \cos ^{2} \phi}{R_{2}}+\frac{k_{1} \cos ^{2} \theta}{R_{1}}-i n^{2} p^{2}\right)
$$

Hence if $R_{1}$ and $R_{2}$ are large $\left|\delta_{2} / \eta_{0}\right| \ll 1$ since $n p \mid k_{1} \gg 1$.

Using the saddle point approximation formula (6.12) we obtain the displacement potential of the wave as:

$$
\phi_{s 2}=\frac{\varepsilon(-2 \pi)^{3 / 2} G_{2}}{\pi B_{1} B_{2} \Delta_{s}^{1 / 2}} \exp \left[-i k_{1} R_{1}-i k_{2} R_{2}+\log (2 \varepsilon)\right]
$$

where $B_{1}, B_{2}, G_{2}$ and $A_{s 1}$ are given by $(8.31)$ $-(8.33)$.

(8.38) is the potential of the PS waves which are reflected from the troughs.

\section{§ 9. Conclusion}

The problem of a line source of compressional type in a halfspace whose free surface is irregular is attacked by the approximate method of small perturbations. We have considered in fair detail only the secondary reflected body waves, $P P$ and $P S$, whose amplitudes are proportional to the depth of the surface corrugations. These represent the first order correction terms due to the uneveness of the free surface. The multiple steepest descent approximation used is unable to bring out such diffracted waves as the Rayleigh waves and in order to investigate these the expressions obtained involving triple integrals will have to be evaluated one at a time by either the Sommerfeld's method of approximation or a combination of that method and the method of steepest descent. This will be reported in another paper.

For a symmetrical curvilinear surface with a single isolated irregularity like a gently sloping ditch in a horizontal plane it is found that if its half-width is large compared with the wavelength of the incident wave the surface scatters the waves as though it were flat and the secondary waves are reflected in the directions of specular reflection. Whereas if the half-width is small compared with the wavelength of the incident wave the secondary reflected waves appear to diverge from the point of intersection of the axis of symmetry of the surface and the horizontal plane asymptotic to it.

In the case of a sinusoidal surface, if the wavelength of the surface is large compared with that of the incident wave the secondary waves are reflected in the specular directions. If the surface wavelength is small compared with that of the wave then the surface behaves as a grating; the secondary waves are reflected from the troughs of the surface.

If the ratio of the half-width or wavelength of the surface to the wavelength of the incident wave is large the amplitudes of the secondary waves are inversely proportional to the square root of the distance between the point of observation and the image of the source in the horizontal plane $z=0$, which is the plane approximating the surface. If the ratio is small the amplitudes are inversely proportional to the square root of the distance from the troughs to the point of observation. 
The deeper the source the smaller the amplitudes of the secondary waves.

\section{Acknowledgments}

The author is grateful to Dr. E. R. LAPwood for illuminating discussions and to $\mathrm{Mr}$. L. LENCHES who prepared the diagrams.

\section{References}

SKURIDIN, G. A.:

1957 Izv. Akad. Nauk (Geofiz) SSSR 2, 36. SATO, R.:

$1955 Z$ Zisin, Ser. 8, 8.
SATO, R.:

1956 Zisin, Ser. 8, 121.

CILBERT, F. and KNOPOFF, L.:

1960 J. Geoph. Res. 65, 3437.

ABUBAKar, I.:

1962 Pro. Camb. Phil. Soc. 58, 136.

RICE, S. O.:

1951 Comm. Pure Appl. Math. 4, 351.

LAMB, H.:

1904 Phil. Trans. Roy. Soc. Lond., A, 203, 1.

LAPWOOD, E. R.:

1949 Phil. Trans. Roy. Soc. Lond., A, 242, 63.

PoL, B. v. d. and BREMMER, H.:

1937 Phil. Mag. 24, 141. 